\title{
Fluid-Fluid Interfacial Effects in Multiphase Flow during Carbonated Waterflooding in Sandstone: Application of X-ray Micro-Computed Tomography and Molecular Dynamics
}

Yongqiang Chen ${ }^{1 *}$, Ahmad Sari² ${ }^{2}$ Junju $\mathrm{Mu}^{1}$, Maxim Lebedev ${ }^{3}$, Ali Saeedi², Vahid J Niasar ${ }^{{ }^{*}}$, Quan $\mathrm{Xie}^{2^{*}}$

1 Department of Chemical Engineering and Analytical Science, The University of Manchester, Manchester, M13 9PL, United Kingdom

${ }^{2}$ Discipline of Petroleum Engineering, WA School of Mines: Minerals, Energy and Chemical Engineering, Curtin University, 26 Dick Perry Avenue, Kensington, Western Australia 6151, Australia

${ }^{3}$ Western Australian School of Mines (WASM): Minerals, Energy and Chemical Engineering, Curtin University, 26 Dick Perry Avenue, Kensington, Western Australia 6151, Australia

*E-mail of Corresponding Authors:

Yongqiang Chen:

yongqiang.chen@manchester.ac.uk

chenyongqiang86@foxmail.com

Vahid J Niasar:

vahid.niasar@manchester.ac.uk

Quan Xie:

quan.xie@curtin.edu.au

To support the observed interfacial phase in Figs. 3, 4 and 5, we provided the following X-ray images at different positions of the samples. The following images clearly shows that the slope of X-ray intensity curves is gentler in carbonated low salinity water than in carbonate high salinity water. The differences in the slope of X-ray curves support the findings in Figs. 3, 4 and 5 and demonstrate the generation of interfacial phase with increasing $\mathrm{CO}_{2}$ amount in the carbonated water.

\section{X-ray intensity distribution after carbonated high salinity waterflooding}



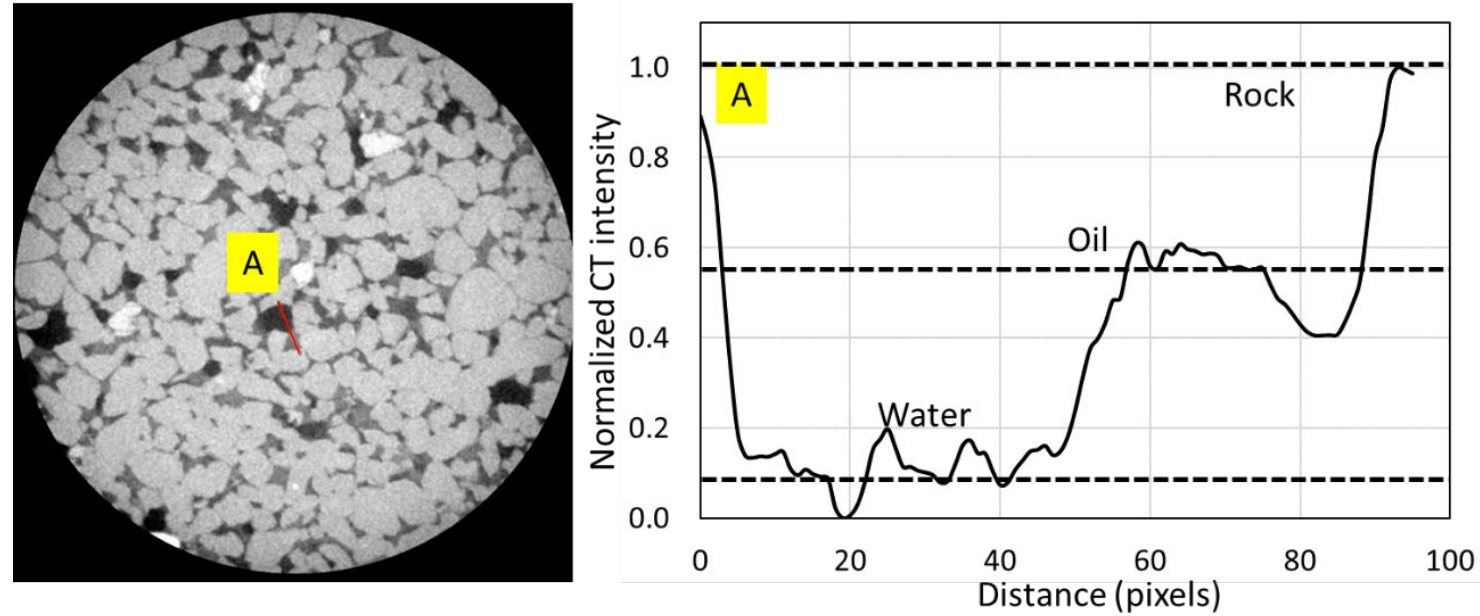

Fig. S1 X-ray intensity distribution after carbonated high salintiy waterflooding at slice No. 200
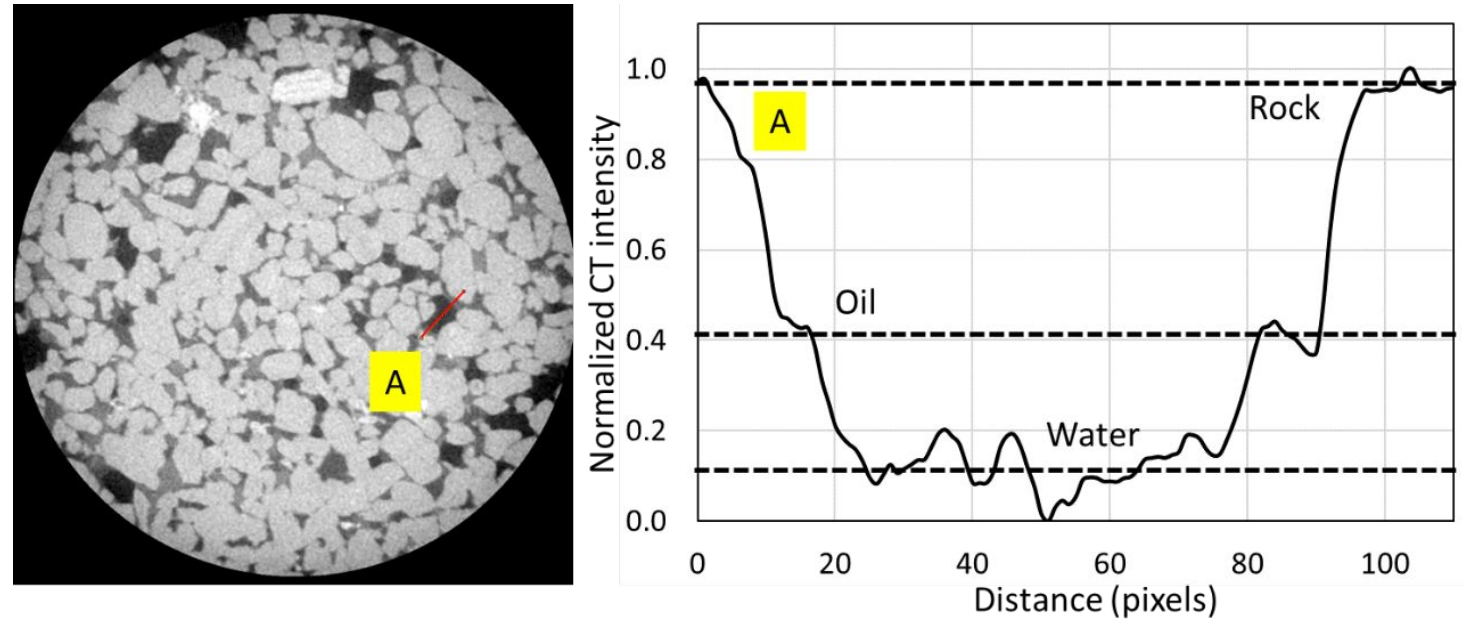

Fig. S2 X-ray intensity distribution after carbonated high salintiy waterflooding at slice No. 300
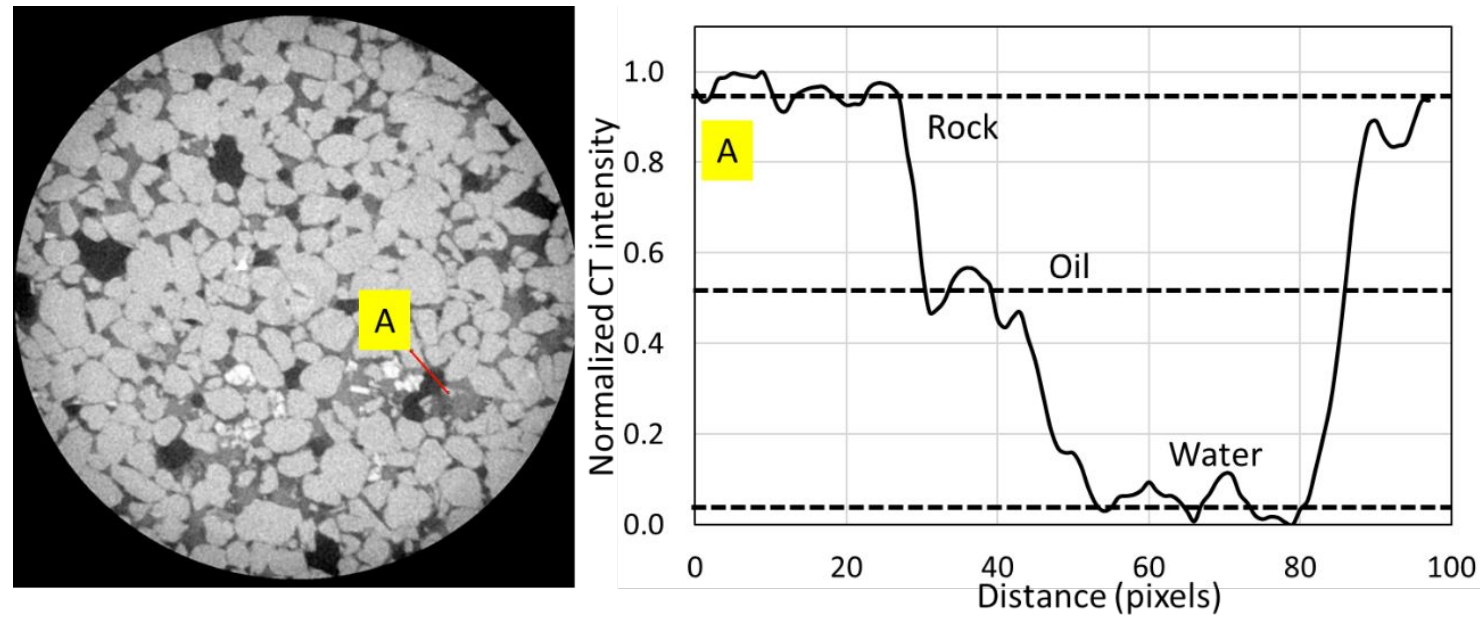

Fig. S3 X-ray intensity distribution after carbonated high salintiy waterflooding at slice No. 400 

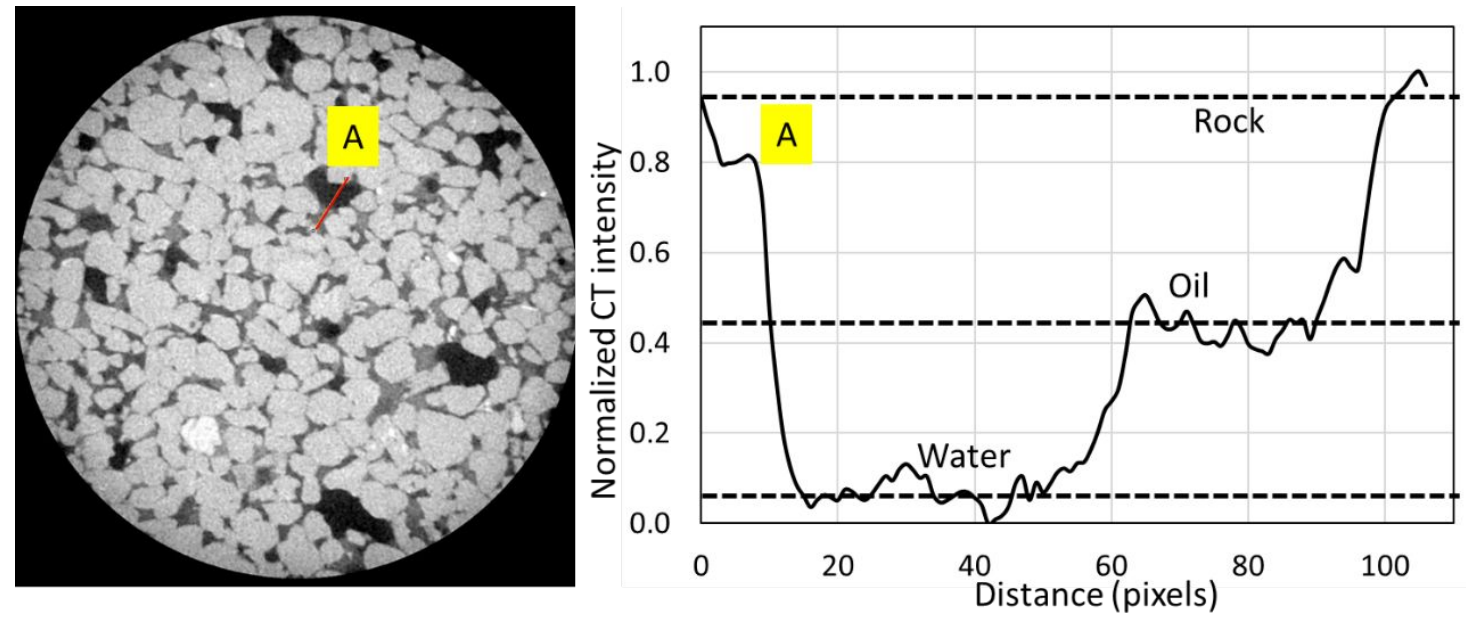

Fig. S4 X-ray intensity distribution after carbonated high salintiy waterflooding at slice No. 500
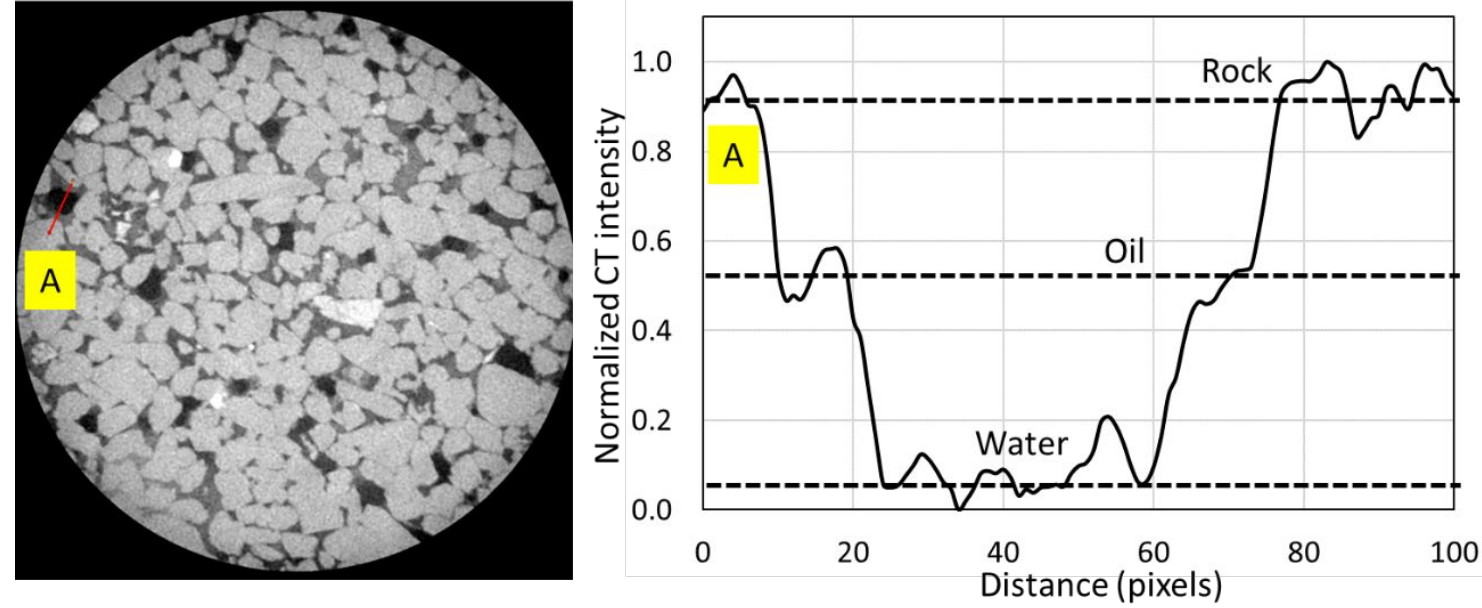

Fig. S5 X-ray intensity distribution after carbonated high salintiy waterflooding at slice No. 600
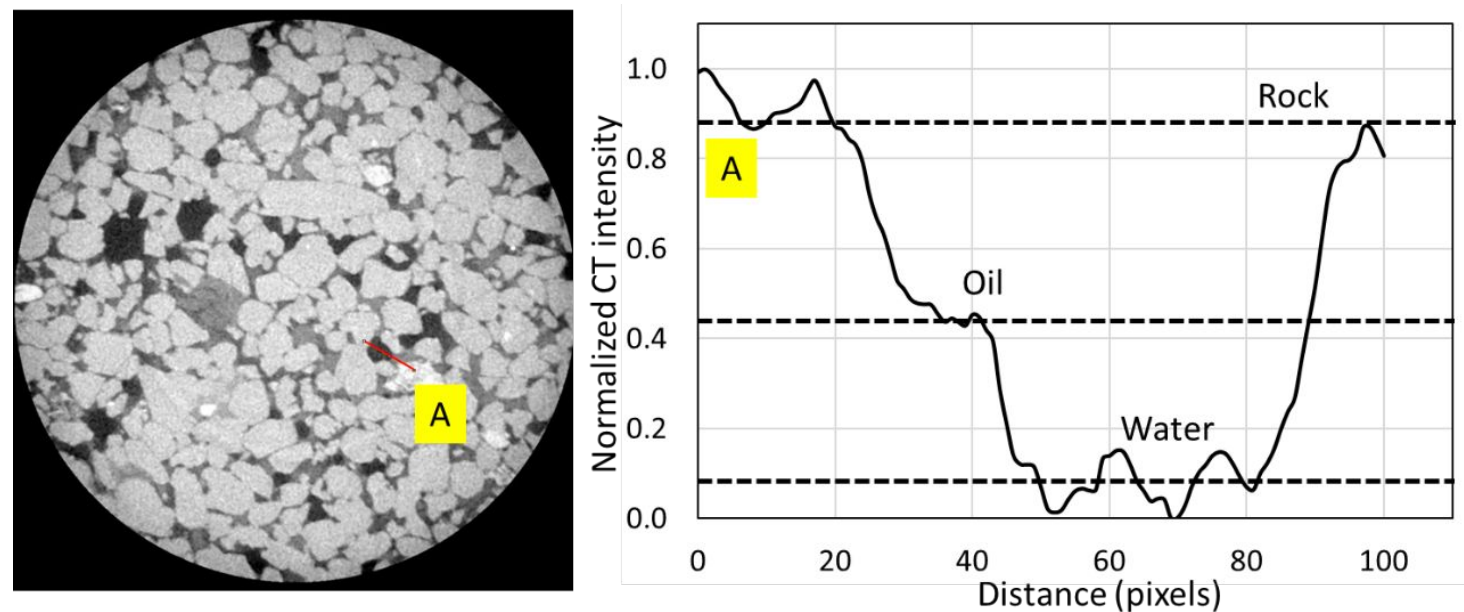

Fig. S6 X-ray intensity distribution after carbonated high salintiy waterflooding at slice No. 700 

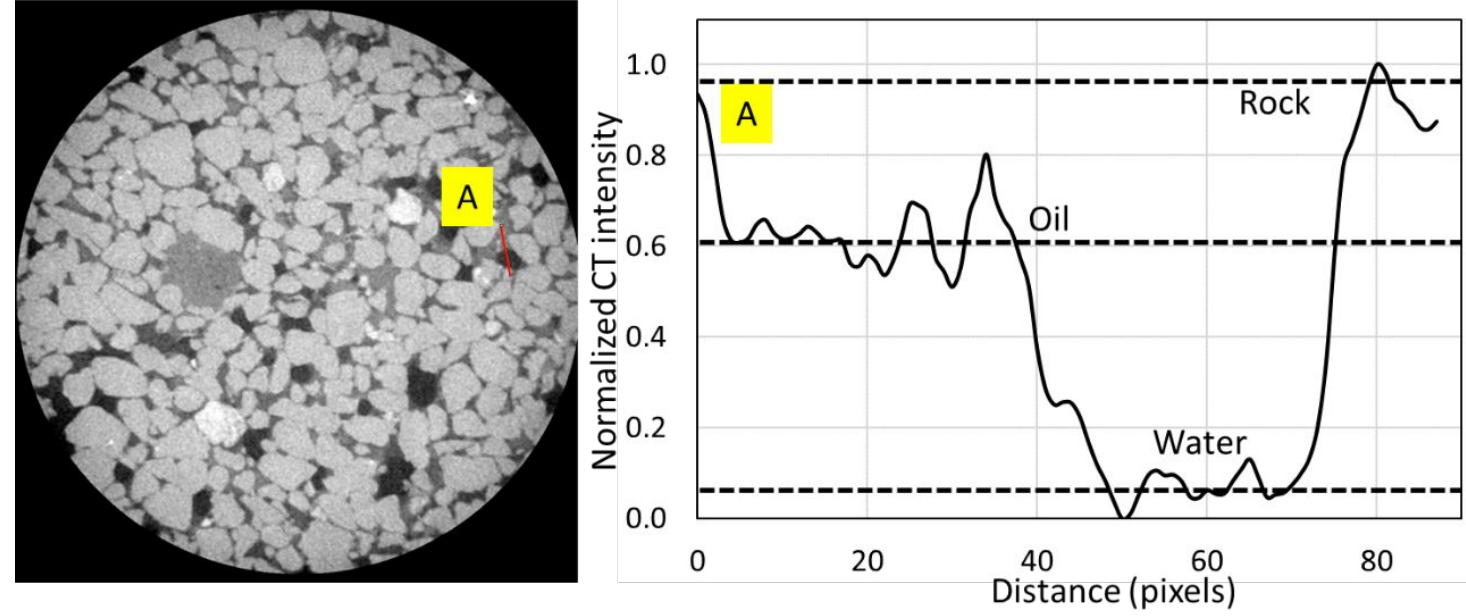

Fig. S7 X-ray intensity distribution after carbonated high salintiy waterflooding at slice No. 800
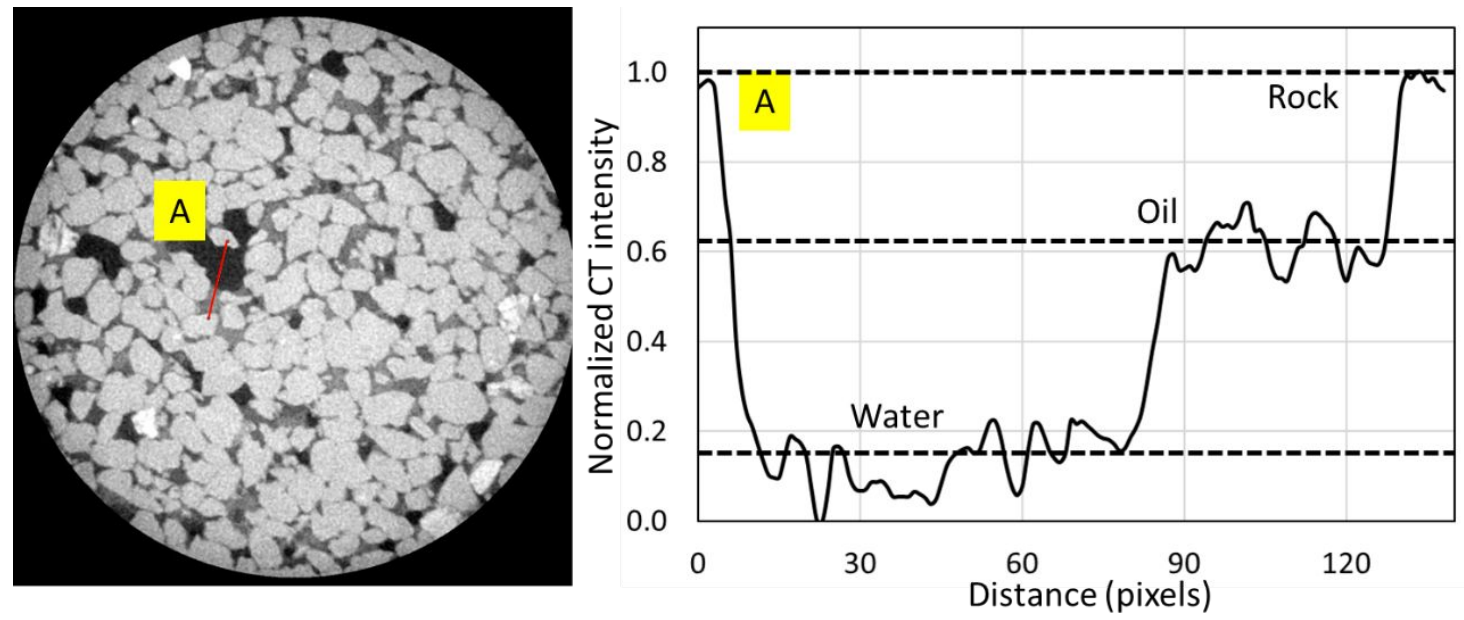

Fig. S8 X-ray intensity distribution after carbonated high salintiy waterflooding at slice No. 900

X-ray intensity distribution after carbonated low salinity waterflooding 

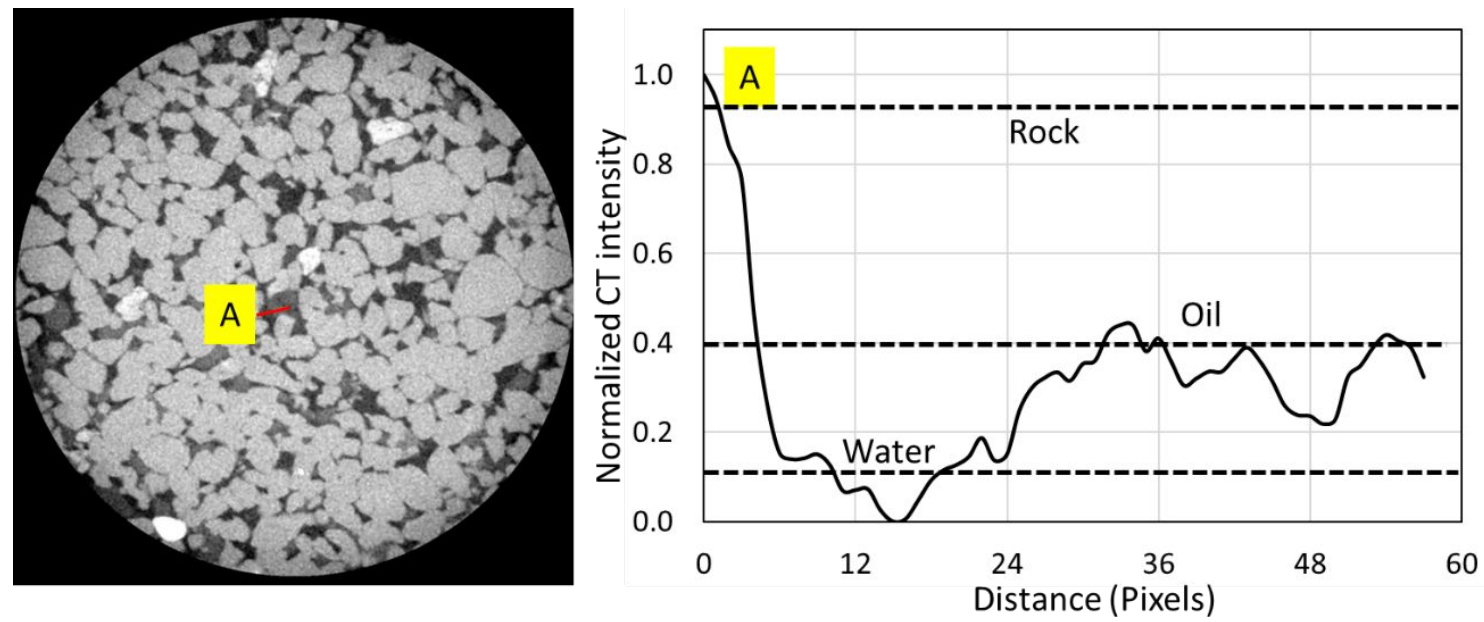

Fig. S9 X-ray intensity distribution after carbonated low salintiy waterflooding at slice No. 200
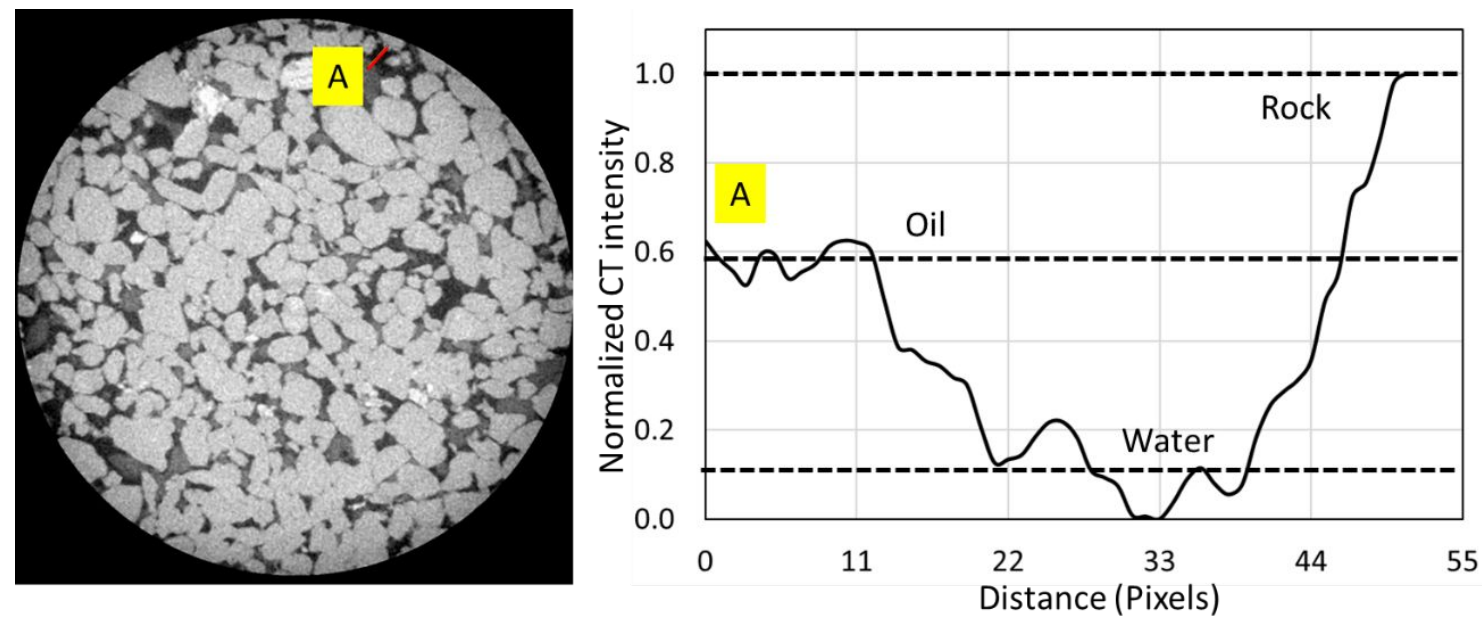

Fig. S10 X-ray intensity distribution after carbonated low salintiy waterflooding at slice No. 300
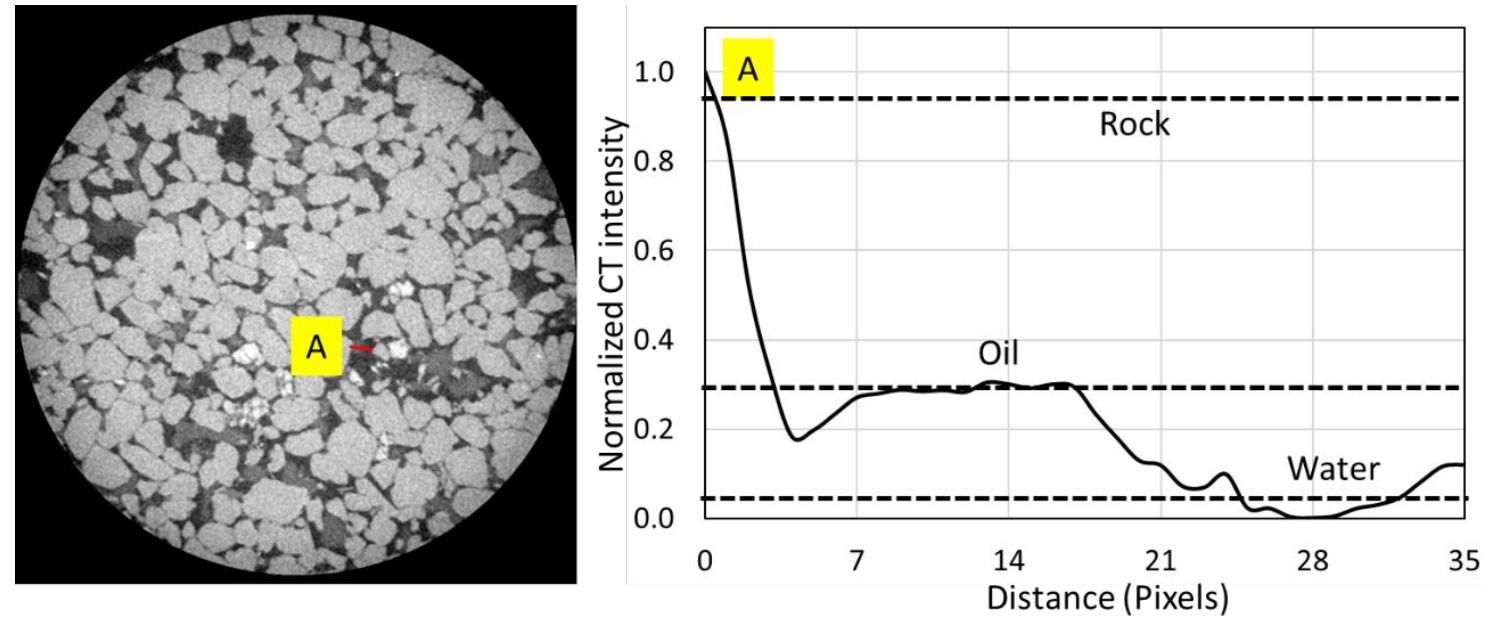

Fig. S11 X-ray intensity distribution after carbonated low salintiy waterflooding at slice No. 400 

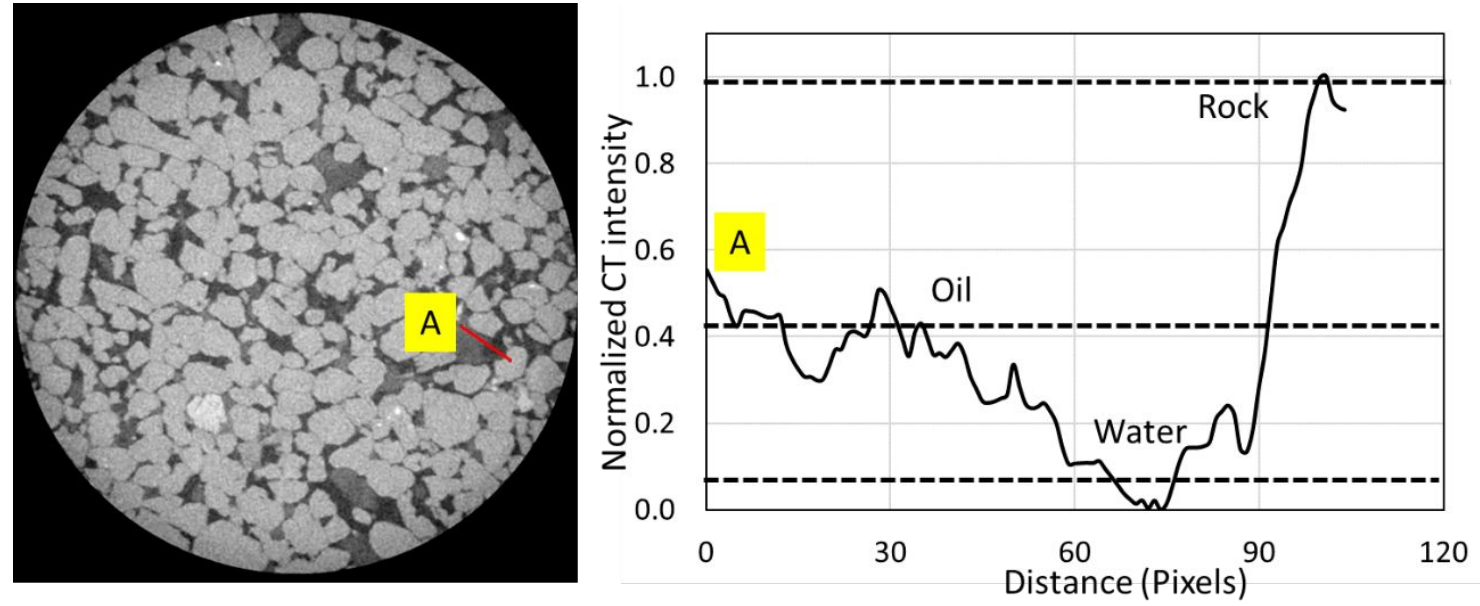

Fig. S12 X-ray intensity distribution after carbonated low salintiy waterflooding at slice No. 500
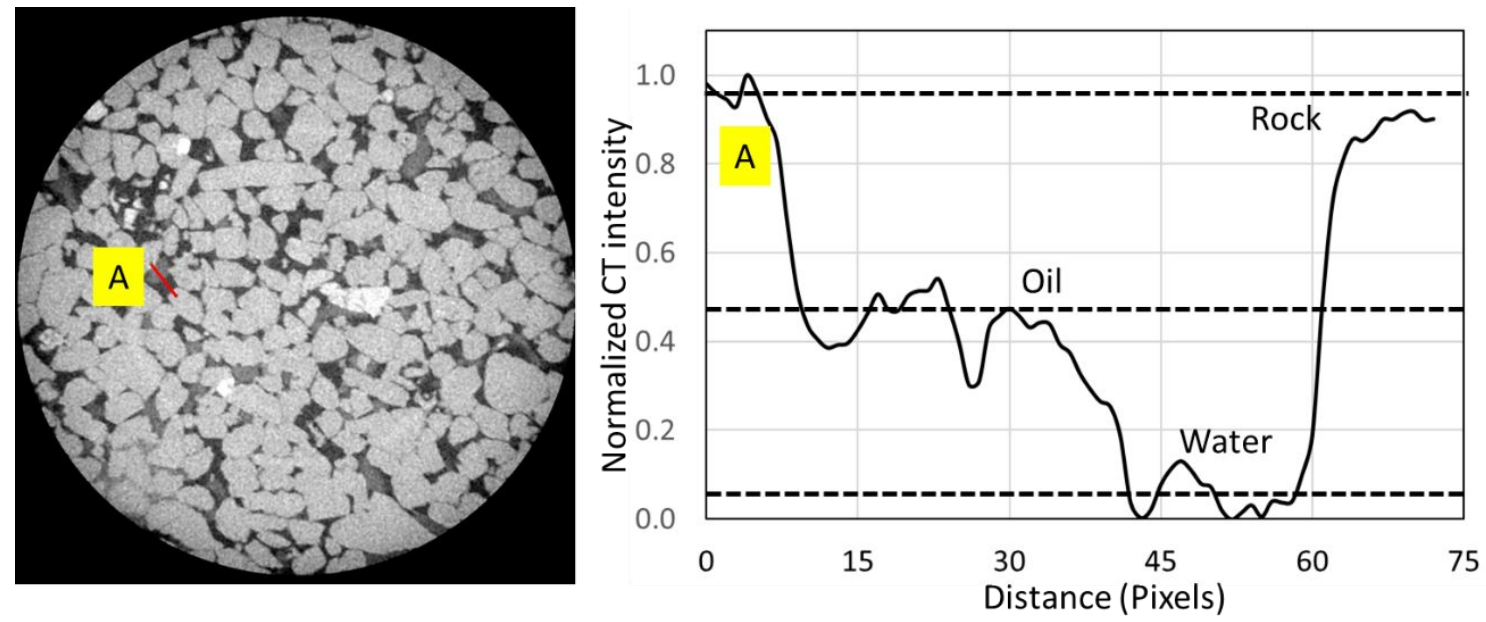

Fig. S13 X-ray intensity distribution after carbonated low salintiy waterflooding at slice No. 600
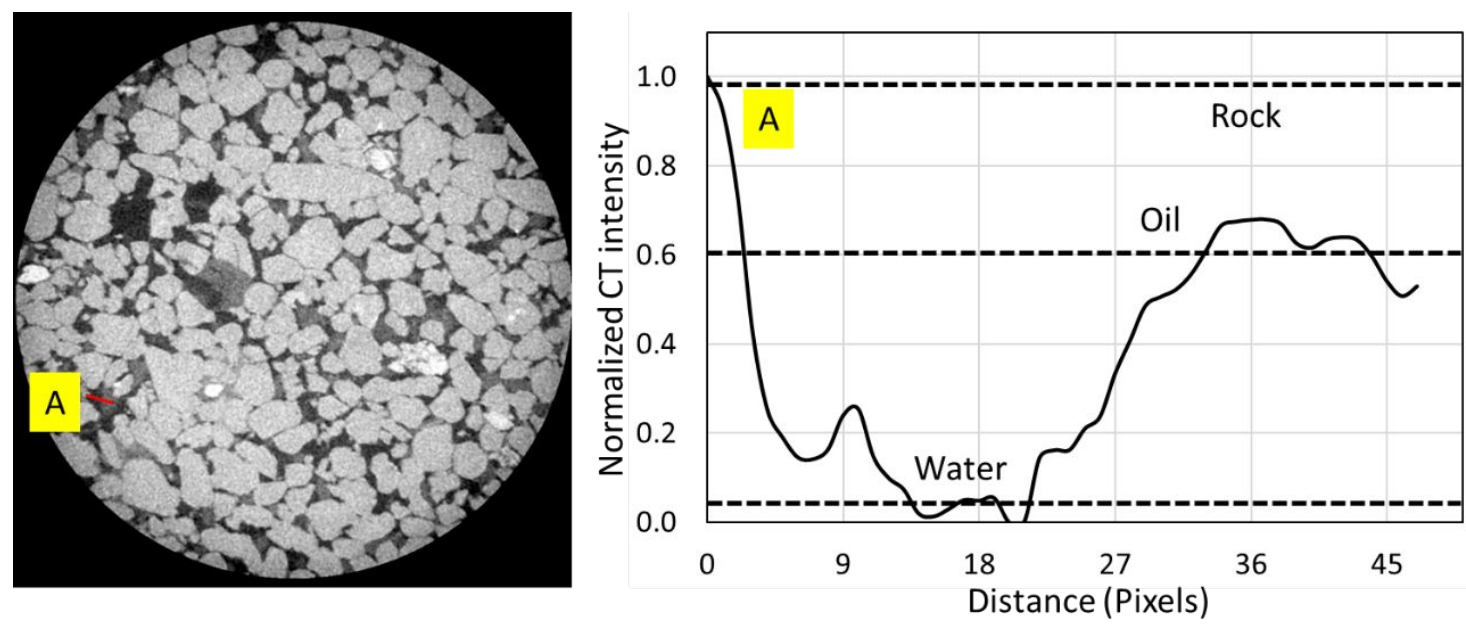

Fig. S14 X-ray intensity distribution after carbonated low salintiy waterflooding at slice No. 700 

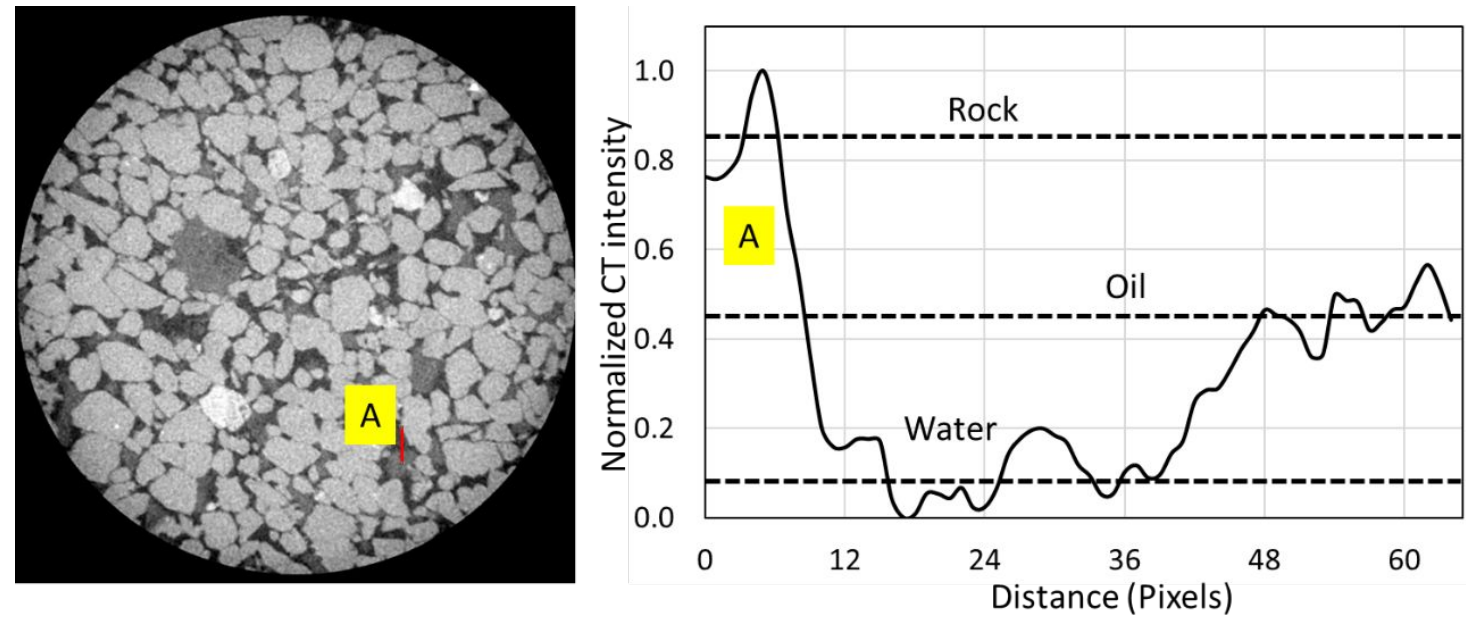

Fig. S15 X-ray intensity distribution after carbonated low salintiy waterflooding at slice No. 800
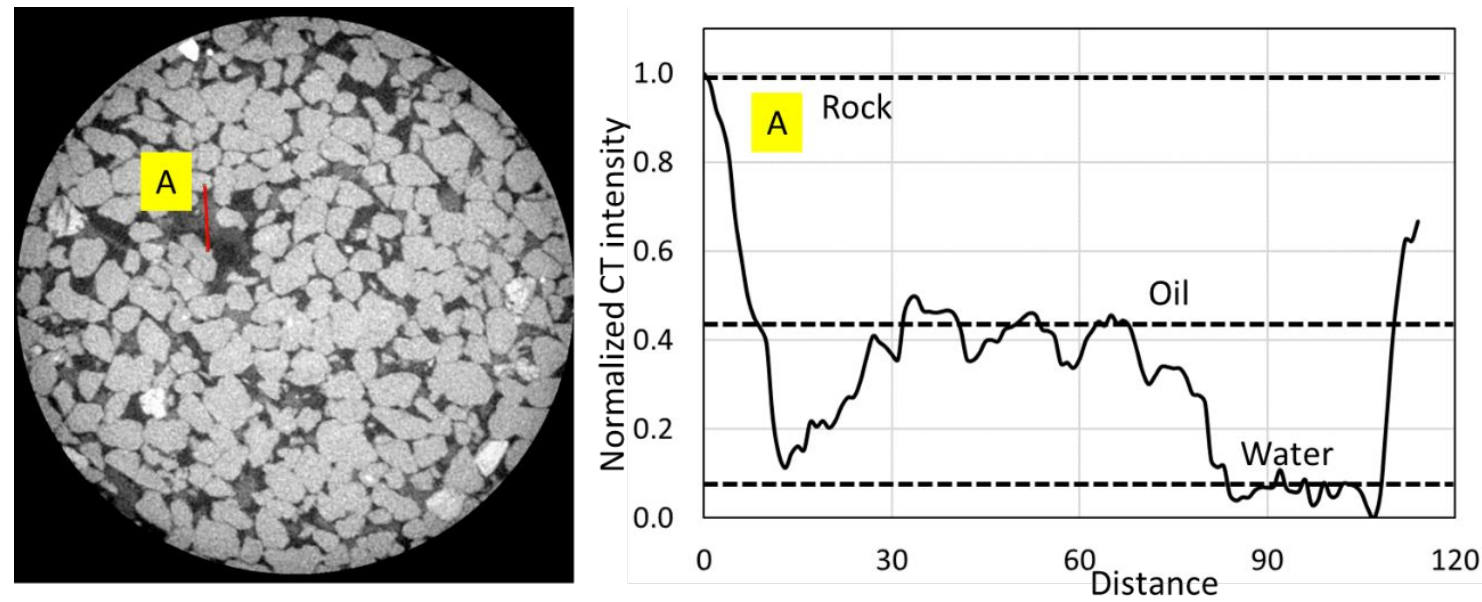

Fig. S16 X-ray intensity distribution after carbonated low salintiy waterflooding at slice No. 900 